\title{
A CHARACTERIZATION OF STANDARD FLAT TORI
}

\author{
BANG-YEN CHEN
}

Abstract. A new characterization of standard flat tori is obtained.

1. Introduction. Let $M$ be a surface immersed in a euclidean 4-space $E^{4}$ and let $\nabla$ and $\nabla^{\prime}$ be the covariant differentiations of $M$ and $E^{4}$ respectively. Let $X$ and $\boldsymbol{Y}$ be two tangent vector fields on $M$. Then the second fundamental form $\boldsymbol{h}$ is given by

$$
\nabla_{X}^{\prime} Y=\nabla_{X} Y+h(X, Y) .
$$

If $\xi$ is a normal vector field on $M$, we write

$$
\nabla_{X}^{\prime} \xi=-A_{\xi}(X)+D_{X} \xi,
$$

where $-A_{\xi}(X)$ and $D_{X} \xi$ denote the tangential and normal components of $\nabla_{X}^{\prime} \xi$. Then we have

$$
\left\langle A_{\xi}(X), \boldsymbol{Y}\right\rangle=\langle\boldsymbol{h}(\boldsymbol{X}, \boldsymbol{Y}), \boldsymbol{\xi}\rangle,
$$

where $\langle$,$\rangle denotes the scalar product of E^{4}$. A normal vector field $\xi$ on $M$ is said to be parallel if $D_{X} \xi=0$ for all tangent vector fields $X$. In the following, a minimal section on $M$ is a unit normal vector field $\xi$ on $M$ with $\operatorname{Tr} A_{\xi}=0$. For a minimal section $\xi$, if $\operatorname{det} A_{\xi} \neq 0$ everywhere, then the minimal section $\xi$ is said to be nondegenerate.

The main purpose of this short note is to prove the following

THEOREM. Let $M$ be a compact surface in $E^{4}$ with Gauss curvature $G \leqq 0($ or $\geqq 0)$. If there exists a parallel nondegenerate minimal section on $M$, then $M$ is a standard flat torus, i.e., $M$ is the product surfaces of two plane circles.

2. Proof of the Theorem. Let $\xi$ be the parallel nondegenerate minimal section on $M$. Then we may choose a local field of orthonormal frame $e_{1}, e_{2}, e_{3}, e_{4}$ in $E^{4}$ over $M$ such that $e_{3}=\xi$ and $e_{1}, e_{2}$ are in the principal directions of $e_{3}$ (and hence $e_{1}, e_{2}$ are tangent to $M$ and $e_{3}, e_{4}$ are normal to

Received by the editors May 2, 1972.

AMS (MOS) subject classifications (1970). Primary 53A05.

Key words and phrases. Standard flat torus, minimal section, nondegenerate, normal vector field, parallel. 
$M)$. Let $h_{i j}^{r}, r=3,4 ; i, j=1,2$, be the coefficients of the second fundamental form $h$. Then we have

$$
A_{e_{3}}=\left(\begin{array}{cc}
a & 0 \\
0 & -a
\end{array}\right), \quad A_{e_{4}}=\left(\begin{array}{ll}
b & e \\
e & c
\end{array}\right) .
$$

Since $e_{3}=\xi$ is nondegenerate and parallel, we may assume $a>0$ everywhere. Let $\omega_{1}$ and $\omega_{2}$ be the dual frame of $e_{1}, e_{2}$. We put

$$
\nabla^{\prime} \boldsymbol{e}_{A}=\sum_{B} \omega_{A B} \boldsymbol{e}_{B}, \quad \omega_{A B}=-\omega_{B A}, \quad A, B, C, \cdots=1,2,3,4 .
$$

Then we have

$$
\begin{aligned}
& \omega_{13}=a \omega_{1}, \quad \omega_{23}=-a \omega_{2}, \\
& \omega_{14}=b \omega_{1}+e \omega_{2}, \quad \omega_{24}=e \omega_{1}+c \omega_{2} . \\
& \omega_{34}=0 .
\end{aligned}
$$

The structure equations are given by

$$
\begin{aligned}
d \omega_{i} & =\sum_{j} \omega_{i j} \wedge \omega_{j}, \quad i, j, k, \cdots=1,2 . \\
d \omega_{A B} & =\sum_{C} \omega_{A C} \wedge \omega_{C B} .
\end{aligned}
$$

The Gauss curvature $G$ is given by

$$
G=\operatorname{det}\left(A_{3}\right)+\operatorname{det}\left(A_{4}\right), \quad A_{e_{3}}=A_{3}, \quad A_{e_{4}}=A_{4} .
$$

Taking exterior differentiations of (1) and applying (3) we obtain

$$
2 a d \omega^{i}+d a \wedge \omega^{i}=0, \quad i=1,2 .
$$

From (7) we can consider local coordinates $(u, v)$ in an open neighborhood $U$ of a point $p \in M$ such that

$$
d s^{2}=E d u^{2}+G d v^{2}, \quad \omega_{1}=E^{1 / 2} d u, \quad \omega_{2}=G^{1 / 2} d v,
$$

where $d s^{2}$ is the first fundamental form and $E$ and $G$ are local positive functions on $U$. From (8) equation (7) becomes

$$
d(a E) \wedge d u=0, \quad d(a G) \wedge d v=0,
$$

which shows that $(a E)$ is a function of $u$ and $(a G)$ is a function of $v$. By making the following coordinate transformation

$$
u^{\prime}=\int(a E)^{1 / 2} d u, \quad v^{\prime}=\int(a G)^{1 / 2} d v
$$


we see that there exists a neighborhood $V$ of each point $p \in M$ such that there exist isothermal coordinates $(u, v)$ in $V$ with

$$
\begin{aligned}
d s^{2} & =f\left\{d u^{2}+d v^{2}\right\}, \quad \omega_{1}=f^{1 / 2} d u, \quad \omega_{2}=f^{1 / 2} d v, \\
a f & =1,
\end{aligned}
$$

where $f=f(u, v)$ is a positive function defined on $V$. It is well known that the Gauss curvature $G$ satisfies

$$
G=-(1 / 2 f) \Delta \log (f),
$$

with respect to the isothermal coordinates $(u, v)$. Hence the condition $G \leqq 0$ (resp. $G \geqq 0$ ) with $a f=1$ implies $\Delta \log (a)=-\Delta \log (f) \leqq 0$ (resp. $\geqq 0$ ). By Hopf's lemma, we see that $\log (a)$ is a constant on $M$. Hence the Gauss curvature satisfies

$$
G=-(1 / 2 f) \Delta \log (f)=(a / 2) \Delta \log (a)=0 .
$$

This implies that

$$
a=\text { constant } \neq 0, \quad G=0 .
$$

On the other hand, by taking exterior differentiation of (3) we obtain $e=0$. Hence, (1) and (2) are reduced to

$$
\begin{gathered}
\omega_{13}=a \omega_{1}, \quad \omega_{23}=-a \omega_{2}, \quad a=\text { constant }, \\
\omega_{14}=b \omega_{1}, \quad \omega_{24}=c \omega_{2}, \quad b c=a^{2}>0 .
\end{gathered}
$$

Taking exterior differentiations of (14) and applying (3), we obtain

$$
d \omega_{1}=\omega_{12} \wedge \omega_{2}=0, \quad d \omega_{2}=-\omega_{12} \wedge \omega_{1}=0 .
$$

These imply that

$$
\omega_{12}=0 \text {. }
$$

On the other hand, by taking exterior differentiations of (15), we obtain

$$
d b \wedge \omega_{1}=d c \wedge \omega_{2}=0 .
$$

Since $b c=a^{2}=$ constant $>0$, we have

$$
(d b) c+b(d c)=0, \quad b c>0 .
$$

Combining (18) and (19) we get

$$
b, c=\text { constants. }
$$


Consequently, the matrix $\left(\omega_{B}^{A}\right)$ is given by

$$
\left(\begin{array}{cccc}
0 & 0 & a \omega_{1} & b \omega_{1} \\
0 & 0 & -a \omega_{2} & c \omega_{2} \\
-a \omega_{1} & a \omega_{2} & 0 & 0 \\
-b \omega_{2} & -c \omega_{2} & 0 & 0
\end{array}\right), \quad a, b, c \text { are nonzero constants. }
$$

Therefore, we may conclude that the surface $M$ is a product surface of two plane circles in $E^{4}$. This completes the proof of the Theorem.

Department of Mathematics, Michigan State University, East Lansing, MiCHIGAN 48823 